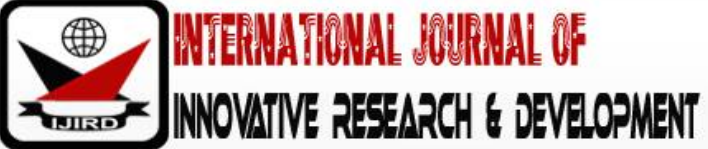

ISSN 2278 - 0211 (Online)

\section{Dietary Intake of Pupils, 8-14 Years in Bungoma County, Kenya}

\author{
Rhoda Loreen Musungu \\ Postgraduate Student, Department of Nutrition Sciences \\ Masinde Muliro University of Science and Technology, Kenya \\ Asenath Sigot \\ Professor, Department of Nutrition Science \\ Masinde Muliro University of Science and Technology, Kenya \\ Dr. Jane Situma \\ Lecturer, Department of Nutrition Sciences \\ Masinde Muliro University of Science and Technology, Kenya
}

\begin{abstract}
:
Good nutrition is crucial for forming proper eating habits to provide the much needed nutrients for growth and development. Moreover, at school age, children develop their nutrition practices which are important in relieving the occurrence of diet related chronic diseases later in life. Globally, malnutrition among school aged children is a public health concern with more than two hundred million children stunted. The objective of this study was to establish the dietary intake of pupils, 8-14 years in Bungoma County, Kenya. The study adopted a descriptive, cross-sectional study design. Multi-stage random sampling was used to determine the schools and pupils, 8-14 years, included in the study. The study population was pupils attending public primary schools in Bungoma County and a total of four hundred pupils were enrolled for the study. Data collection tools used included questionnaires for quantitative data collection. Data was collected on 24 hour dietary recall and food frequency questionnaire. Data collected was analyzed using and the Statistical Package for Social Sciences (SPSS) version 20 to be able to categorize the pupils appropriately. Data analyzed was presented using tables, pie charts and bar graphs. The study found out that majority of the pupils had low dietary diversity $(n=270,67.50 \%)$ and had inadequate dietary intake $(n=217,54.3 \%)$. The mean kilocalorie consumption was 2217.63 kilocalories. Adequate dietary intake is necessary for pupils to realize their full potential in life. Nutrition education on dietary intakes should begin at home and also be integrated in the pupils' school curriculum.
\end{abstract}

Keywords: Dietary Intake, pupils, 8-14 years

\section{Introduction}

School age is a unique time of development and improvement, framing a solid establishment for good wellbeing and a profitable grown-up life. It is also known that dietary intake provides the much needed nutrients for growth, long term health, cognition and educational achievement. A child's eating patterns and dietary intake might influence their growth especially in SAC (Grosver \& Smolin, (2009). Thus, the dietary intake of a child must supply the nutrients that are needed for their growth and development, and also for body maintenance and energy for physical activities (Sophie and Peninah, 2014). Adequate nutrition is a right all children have, which is essential for the attainment of the highest standards of health (United Nations Children's Fund (UNICEF), 2016). Hunger and malnutrition among children in developing countries continues to impair their health, quality of life and survival (FAO, 2011). Malnutrition in this group can be prevented as school age offers a window of opportunity to inculcate healthy eating habits that can mitigate the occurrence of diet-related chronic diseases and they can also exhibit catch-up growth. Early interventions will improve their overall development, resulting in better educational outcomes and more productive adults (SCN, 2010; Sophie and Peninah, 2014). Sixty six million primary SAC attend class hungry daily across the developing world with twenty three million of them in Africa (World Food Program (WFP), 2015. Nutritional inadequacies influence children's health, putting them at risk of chronic diseases in adulthood. Also, nutrition deprivation during this critical period constrains the physical and cognitive development of SAC, limiting their educational achievements and social development (Best et al., 2010).

\section{Literature Review}

Inadequate dietary intake is secondary to insufficient or inappropriate food supplies. In some areas, cultural and religious food customs may affect dietary intake (UNICEF, 2016). A study done in Kajiado, Kenya reported that cultural beliefs, taboos and attitudes negatively affected the dietary practices of SAC. Boys considered nutrition as a female domain 
and instead preferred masculine activities to nutrition related activities. Attitudes towards consumption of certain foods were influenced by the school set up; rural or urban. Girls on the other hand, did not consume animal products like eggs and meats as they assumed it will be difficult for them to get married (Mbithe, Kimiywe and Waudo, 2012). It is important to note that SAC tend to eat differently as they did as children due to such factors and also the need for independence and acceptance by their peers, mobility and time spent in school and the pre-occupation with their self-image. To enhance cognition as well as the physical development of pupils, a good amount and quality of food is required (Barooh, 2012).

Another important aspect of dietary intake is dietary diversity. According to a study done by Mukherjee et al., (2017), the dietary intake of school aged children in developing countries is lacking in diversity. Fruits and vegetables have a key role in nutritional balance. They are the main food sources of fiber, vitamins, minerals and other biological components for the growth of the organism (Best et al., 2010). According to the FAO Family Nutrition Guide, the frequency of eating for older children including SAC should be 3 meals and 3 snacks daily (FAO, 2004a). WHO/ FAO dietary guidelines (2010), states that there are important components that ensure adequate dietary intake for optimal nutrition among SAC. These include proteins, especially from animal sources, carbohydrates and fats. In addition to that, micronutrient rich foods should also be included in their diets, with adequate consumption of fruits and vegetables encouraged. Foods rich in iron, folate and vitamin B12 should be in plenty to avoid the occurrence of anemia as it negatively impacts the work capacity, intellectual performance and cognitive development. Sufficient iodine and zinc are also important to optimize mental development and prevent goiter. Vitamin A also plays a critical role in the diets of SAC eye health and immune function.

Meal patterns and food choices are also key in the dietary intake of SAC. Breakfast is the most important meal of the day and should provide most energy needed by the SAC for their learning and school activities. Despite this knowledge, various studies have reported that most SAC miss out on breakfast with its contribution to the energy intake being $10 \%$ of the recommended daily allowance (Mwaniki and Makokha, 2013, Abrahams et al., 2011 and Doku et al., 2013). This was found to occur mostly in the rural populations (Sophie and Peninah, 2014). With regards to food choices, it is important to note that nutrition knowledge among SAC is key for them to establish proper foods required for their growth and development, especially when they eat away from home. A study done by Kiragu et al., (2015), among primary school children in Nairobi found that the pupils had moderate nutrition knowledge and majority of them did not care about what they ate and had a bad attitude towards their diets. Another important issue of concern is the increased consumption of processed foods among SAC. Foods high in sugar, salt and saturated fat are preferred by these children and school canteens stock these foods thus encouraging their consumption (Gharib and Rashid, 2011). This shift is intensified by the rapid replacement of traditional diets with western diets. This has encouraged the consumption of energy dense foods. These foods are mainly eaten away from home and they make a large contribution to the overall diet of the SAC (Nago et al., 2010; Pangani et al., 2016). This study therefore sought to determine various aspects of dietary intake of pupils 8-14 years in Bungoma County, Kenya including the number of meals and snacks consumed in a day, their caloric intake, nutrient adequacy, food frequency consumption of various food groups and their dietary diversity.

\section{Materials and Methods}

The study was conducted in Bungoma County. It has a high child population with 0-14 year olds, being $49 \%$ of the total population. The 2009 population census put the county's population at 1,374,627 (671,222 male; 703,405 female). The County experiences two rainy seasons, the long rains - March to July and short rains August to October. Agriculture is the main economic activity in Bungoma County. Main crops produced include maize, beans, finger millet, sweet potatoes, bananas, Irish potatoes and assorted vegetables. Sugar cane, cotton, palm oil, coffee, sun flower and tobacco are grown as cash crops in the County (Bungoma County Development Plan, 2013). This study adopted a descriptive cross-sectional design, employing quantitative techniques for data collection. This design allows data collection at a point in time. It also describes the characteristics of the sample being studied and can generalize the findings from the sample to a larger population. Pupils attending public primary schools in Bungoma South sub-county, between 8-14 years and whose parents have undertaken consent for them were included in the study.

Multistage random sampling method was used. Four (4) wards out of the 8 wards (Bukembe West, Bukembe East, Township, Khalaba, Musikoma, East Sangalo, West Sangalo, Marakaru/ Tuuti) were randomly selected in the first stage. In the second stage, one public primary school from each selected ward was randomly selected. Two school selected were from the urban region of the sub-county while the other two were from the rural regions of the sub-county.

In the third stage, sampling of students within the four schools was done. Simple random sampling was used to select pupils, aged 8-14 years, who are from classes 4-8, to comprise the desired sample size. First, the researcher identified class 4-8 pupils, 8-14 years from each school and got their total number. Yamane Method (1967) was used to determine the sample size of the study. 400 pupils comprised the sample size of the study.

\section{Results}

Results of the study also found that $50.8 \%$, $(n=203)$ of the pupils were male whereas $49.2 \%(n=197)$ were female. The number of meals consumed in a day by the pupils was determined using a 24 hour dietary recall. A big proportion of the pupils had 3 meals in a day $(n=351,87.80 \%)$ with $0.8 \%(n=3)$ having one meal a day. This is illustrated in Figure 1 below. 


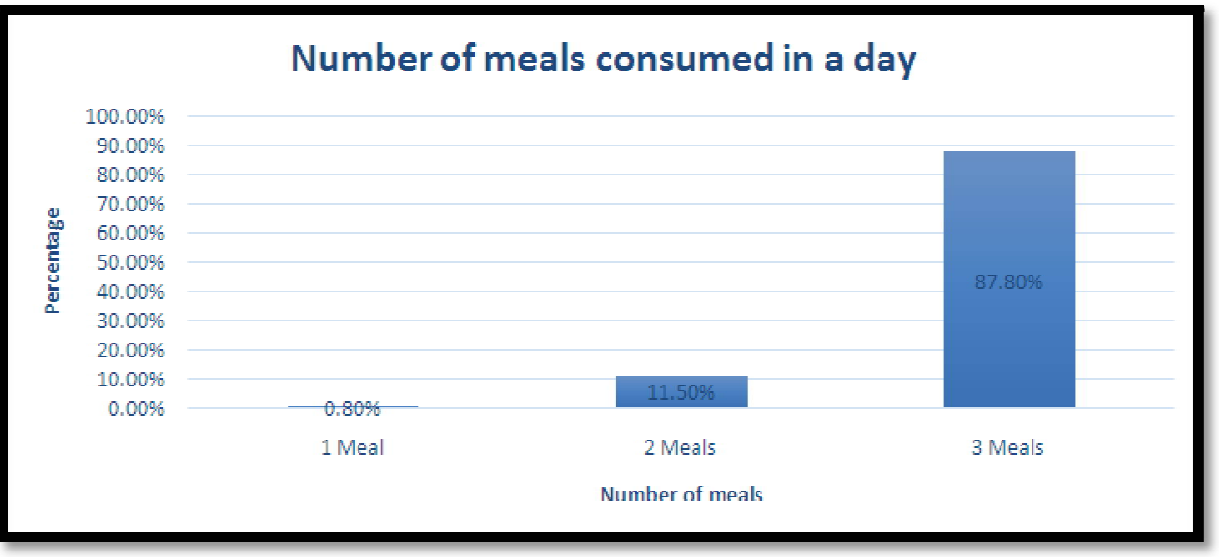

Figure 1: Numbers of Meals Consumed by the Pupils In A Day

On the consumption of snacks, the 24 hour recall was used. Majority of the pupils ate just one snack in a day ( $\mathrm{n}=$ $169,42.3 \%)$, with $3.3 \%(n=13)$ eating 3 snacks in a day. A considerable percentage, ( $n=141,35.3 \%)$ did not have any snack accompanying their meals in a day. The findings are as illustrated in Figure 2 below.

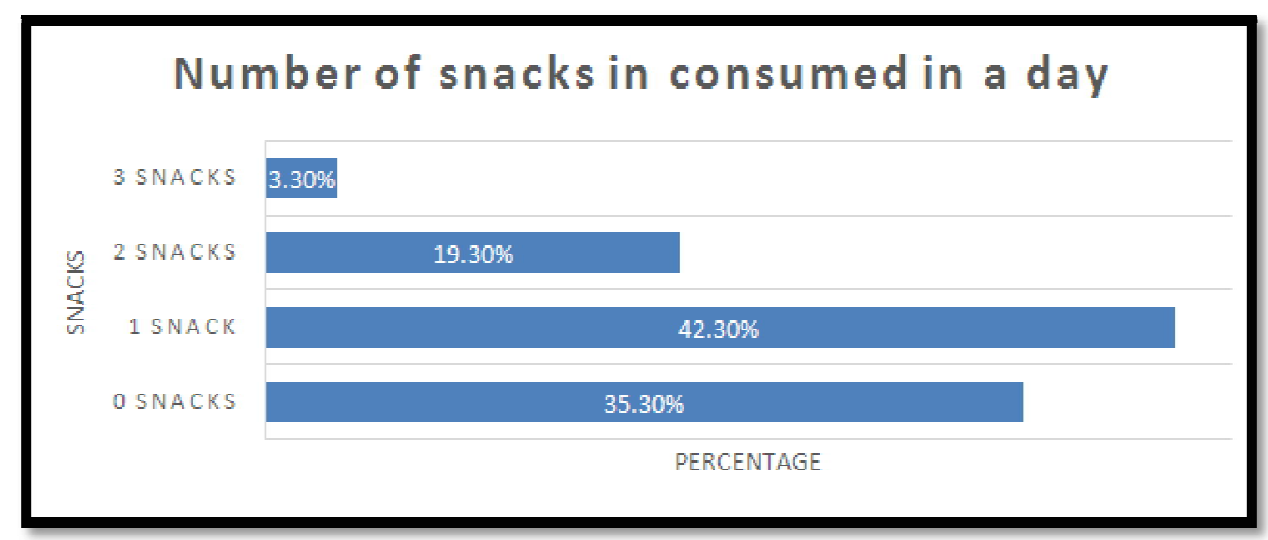

Figure 2: Number of Snacks Consumed by Pupils in a Day

The survey data also revealed the total kilocalorie of the pupils in a day from the 24 hour recall. Using the calculations of the quantities of the meals and snacks consumed according to the Photographic Food Atlas for Kenyan Adolescents (2018) and the Kenya National Clinical Nutrition Manual (2010) Exchanges for carbohydrates, proteins, fats and energy values, the amount of total kilocalories consumed in a day was computed. The findings from the computations were compared to the Recommended Dietary Allowances for school going children which are 2,500 Kcal/ day for males and 2,300 Kcal for females, according to WHO/ FAO, (2010). The mean kilocalorie consumption among the pupils was 2,217.63 Kilocalories in a day with the mean consumption for male pupils being 2,206.26 Kilocalories in a day and for female pupils 2,229.35 Kilocalories in a day. Table 4.4 gives a summary of the findings.

\begin{tabular}{|c|c|c|c|c|}
\hline Gender & Mean & N & Std. Deviation & \% of Total Sum \\
\hline Male & $2,206.26$ & 203 & 490.551 & $50.5 \%$ \\
\hline Female & $2,229.35$ & 197 & 568.913 & $49.5 \%$ \\
\hline Total & $2,217.63$ & 400 & 530.051 & $100.0 \%$ \\
\hline
\end{tabular}

Table 1: Mean Kilocalorie Intake of Pupils in a day

Nutrient adequacy was another important component of dietary intake which the study looked at. The nutrient adequacy was compared to the recommended standards by WHO/ FAO (2010) of 2,500 Kcal/ day for male pupils and 2,300 $\mathrm{Kcal} /$ day for female pupils of this age group. Findings from this study indicated that, of the 400 respondents, almost half of them had inadequate dietary intake $(n=217,54.3 \%)$, with only $45.8 \%(n=183)$ having adequate dietary intake in a day as recommended for their age group. Figure 3 illustrates this. 
NUTRIENT ADEQUACY IN A DAY

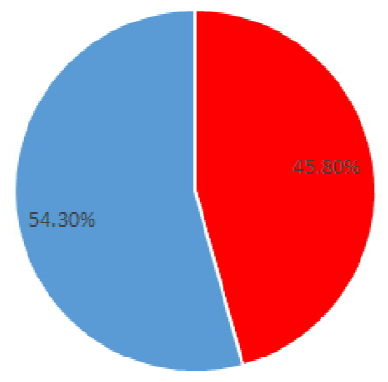

- Adequate Inadequte

Figure 3: Nutrient Adequacy of Pupils in a Day

The comparison of nutrient adequacy among the pupils, 8-14 years in Bungoma County showed that most female pupils $(n=128,63 \%)$, had adequate nutrient intake in a day compared to their male counterparts at $(n=75,37 \%)$. On the other hand, most male pupils had inadequate diets in a day at 55\% $(n=108)$ compared to their female counterparts $(n=89$, $45 \%$ ). This is illustrated in summary on table 4.5 below.

\begin{tabular}{|c|c|c|c|c|c|}
\hline Dietary Intake & Males & Percentage & Females & Percentage & Total \\
\hline Adequate & 75 & $37 \%$ & 128 & $63 \%$ & 203 \\
\hline Inadequate & 108 & $55 \%$ & 89 & $45 \%$ & 197 \\
\hline Total & 183 & & 217 & & 400 \\
\hline
\end{tabular}

Table 2: Comparison of Nutrient Adequacy among Male and Female Pupils

The study also determined the frequency of consumption of different foods in the seven days prior to the survey. The foods were later categorized into food groups of: cereals, meats, eggs, milk and milk products; legumes, nuts and pulses; green leafy vegetables, other vegetables; vitamin A rich fruits and other fruits, roots and tubers and oils and fats as shown in Figure 4. In general, the pupils consumed from all the food groups frequently within the past week preceding the study. Cereals were the most consumed food group $(n=398)$ followed by oils and fats $(n=396)$ and vegetables $(n=389)$ in a week. This could be attributed to the rainy season that was at the time of the study. The least consumed food groups in terms of frequency of consumption in a week were meats $(n=192)$ and roots and tubers $(n=191)$.

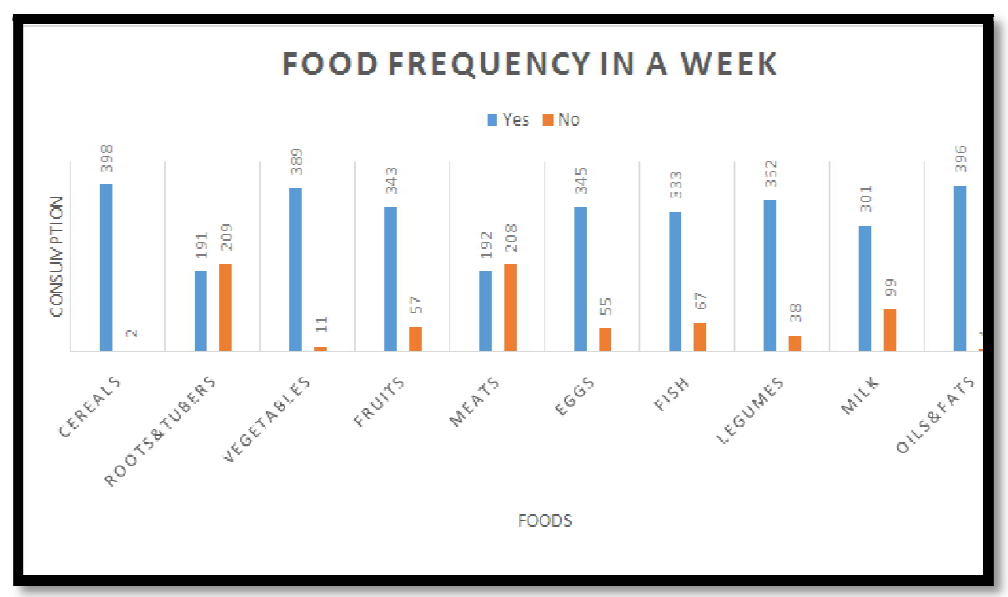

Figure 4: Frequency of Food Group Consumption in a Week

Using the 24 hour recall, the individual dietary diversity score of the pupils was calculated. This is a measure usually done on children over a period of 24 hours. The number of food groups looked at were 10 and dietary diversity scores given were; low dietary diversity for a score of 1 - 4 food groups, medium dietary diversity for a score of 5 - 7 food groups and high dietary diversity for a score of $8-10$ food groups. Majority of the pupils had low individual dietary diversity scores at $67.5 \%(\mathrm{n}=270)$ and $0.8 \%(\mathrm{n}=3)$ having a high dietary individual dietary diversity scores. Figure 4.5 illustrates this. 


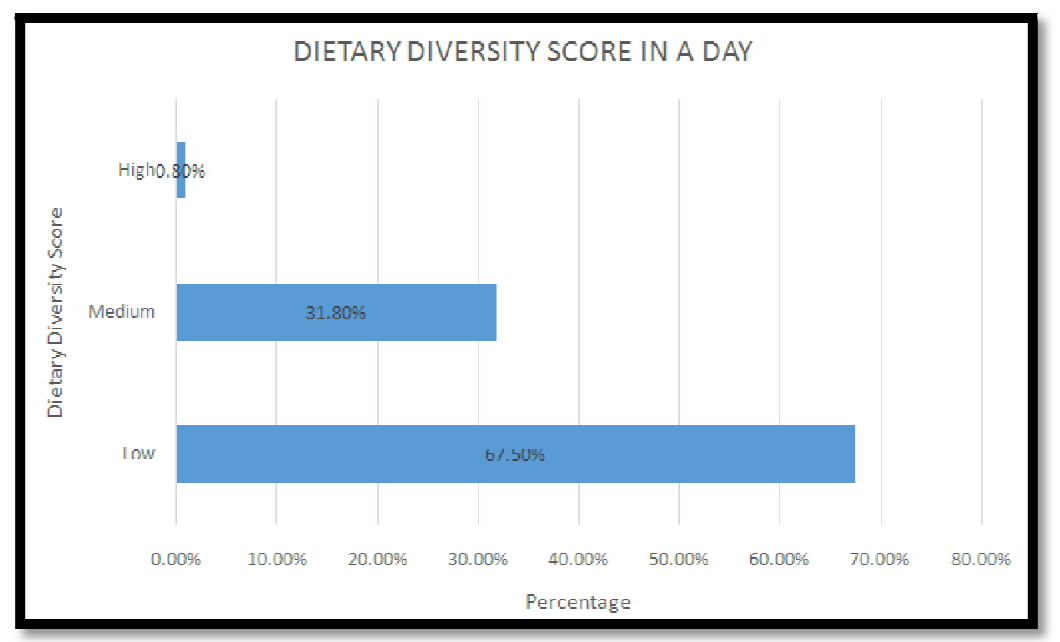

Figure 5: Individual Dietary Diversity Score of Pupils in a Day

\section{Discussion}

School age is an active growing phase and the dietary intake of a child must supply the nutrients needed for a child's growth, development, body maintenance and physical activities. There are important components that ensure adequate dietary intake in order to ensure optimal nutrition among pupils. These include proteins, carbohydrates, fats and micronutrients from fruits and vegetables. This can only be achieved by consuming diverse diets of at least 3 meals and 3 snacks a day (Rajabium, 2009). Also, according to FAO Family Nutrition Guide, the frequency of eating for older children including pupils should be 3 meals and 3 snacks daily (FAO, 2004a). Findings from this study revealed that a big proportion of the pupils had 3 meals in a day as is recommended. However, when it came to the consumption of snacks in a day, majority of them had only one snack which is against the recommended intake of three sacks in a day.

Between the ages of 8-14 years, pupils are in an active growing phase. Their dietary intake greatly influences their growth thus it must supply all the nutrients needed for their growth, development and also for body maintenance and enabling the body's physical activities. Findings from this study observed that almost half of the pupils had inadequate dietary intakes. As was noted by Bandura (2006), insufficient intake of energy may occur because of certain factors, the most common being inadequate monetary resources to purchase food, restrictive dieting or chronic illness. Findings from this study indicated that majority of the respondents did not meet their daily caloric intake with reference to the Recommended Dietary Allowances by WHO/FAO (2010) of $2500 \mathrm{Kcal} /$ day for the male pupils and $2300 \mathrm{Kcal} /$ day for the female pupils. This could partly explain why the pupils had inadequate daily dietary intakes. Similar findings were observed by El-Kassas and Ziade, (2017) on their study on the dual burden of malnutrition and associated dietary lifestyle habits among Lebanese SAC living in orphanages in North Lebanon, that found about half of their study participants had inadequate dietary intakes. This is best explained by the evidence of the pupils' failure to consume an adequate number of meals and snacks a day.

Findings from this study also differ with those done by Opini (2017), on the nutrition status and associated factors among school children aged 4-10 years in Kisii Central, Kisii County which found out that the school children had adequate dietary intakes with at least 4 meals in a day as most foods were available both from own production $53.7 \%$ and through purchase $41.0 \%$. Most children had high consumption of vegetables $34.0 \%$ and cereals $32.0 \%$, foods rich in vitamins and carbohydrates. However findings from this study showed that all the pupils purchased their own meals during lunch time with the average cost of food being 30 shillings for a meal (rice with green grams, githeri and chapati/ mandazi) and 10-20 shillings for snacks (sweets, ice cream and samosas). Breakfast and dinner were being prepared at home where food was purchased by majority of the respondents with only a few being from their own production in their farms.

$54.3 \%$ of the pupils had adequate kilocalorie consumption in a day. Majority of these pupils were from the urban area of the sub-county. This adequate intake was attributed to the consumption of carbohydrate rich foods during their main meals especially during breakfast and dinner and fried, high fat foods during their lunch and snack times. Findings from this study concurred with those done by Gharib and Rashid (2011), on the energy and macronutrient intake and dietary pattern among school children in Bahrain, which noted a shift in diets with rapid replacement of traditional diets with western diets which encourage the consumption of energy dense foods. The analysis of data also revealed that majority of the respondents had a low individual dietary diversity score of 4 or less food groups a day required in the normal diet. This greatly contributed to the nutrient inadequacy of the pupils' dietary intake as the dietary diversity reflects the nutrient quality of ones' diet (FAO, 2011).

Mukherjee et al., (2017) in a study on the dietary habits of school children in Pune City, Maharashtra India, found that the dietary intake of school age children in developing countries is lacking in diversity. Findings from this study concur to their study as most foods consumed were carbohydrate rich with vegetables, fruits and meats being rarely consumed in a day. Similar findings were observed in a study on nutrition status and associated factors among children in public primary schools in Dagoretti, Kenya that found out that few children consumed less than four food groups in a day (Mwaniki and Makokha, 2013). This was as a result of the preference of more carbohydrate rich foods to protein and vitamin rich foods. 
Another important finding was the increased consumption of processed foods which has been intensified by the rapid replacement of traditional foods with western diets. Majority of the pupils in the study preferred processed foods as part of their snacks and these foods made a large contribution to their overall diets. This findings concur to a study done by Granner et al., (2004), on factors of fruit and vegetable intake by race, gender and age among young adolescents which concluded that SAC are consuming plenty of junk foods as their snacks. A similar trend of these eating habits have been observed among pupils in developing countries as was noted by Lazzeri et al., (2013) and Pangani et al., (2016) in Tanzania. This was due to their readily availability in both study areas. This is a growing area of concern among the school aged population that must be looked into keenly as these foods are mainly eaten away from home and they make a large contribution to the overall diets of SAC.

Doku et al., (2013), in a study done in Ghana on the socio-economic differences in adolescents' breakfast eating, fruit and vegetable consumption and physical activity, noted that fruits and vegetables were rarely eaten by school aged children. Findings from this study however, do not relate with their study as the consumption of fruits and vegetables was quiet high among the food groups consumed during the week prior to the study. This can be attributed to the readily availability of fruits and vegetables in the area as the study was conducted during the rainy season when fruits and vegetables were in plenty. Also, the study area is a cosmopolitan area with plenty of food stuffs especially fruits from neighboring counties of Kakamega and Trans Nzoia and the neighboring country of Uganda. Moreover, Rauber, Hoffman and Vitolo (2014) and Sophie and Peninah (2014) in their studies noted that the consumption of plant based diets from cereals, tubers and roots was high in school aged children in rural populations. This is similar to the findings of this study that recorded a high consumption of plant based foods with cereals (100\%), vegetables (97\%) and legumes (90\%) being mostly consumed compared to meat products (47\%).

An association between school lunches containing animal source foods that increased the intake of micronutrients and had a positive effect on cognitive development, body composition and growth was demonstrated in Kenya by Omwami, Neumann and Bwibo (2011) in their study on the effects of a school feeding intervention on school attendance rates among elementary school children in rural Kenya. Results from this study however indicated poor meats consumption by the pupils in a week with the frequency of meat consumption being the least of all the food groups consumed at $48.3 \%$. This is partly attributed to the low socio-economic status of the caregivers of the pupils thus they cannot purchase the meats and meat products. Similar findings were observed in Benin in a study done by Mitchikpe et al., (2009) on seasonal variation in food pattern but not in energy and nutrient intake of rural Beninese SAC where animal products contributed only $7 \%$ of daily protein intake.

With regards to the meal patterns, it was noted from the study that a big proportion of the pupils consumed three meals in a day that comprised of breakfast, lunch and dinner with only a few of them not taking three meals. Findings from this study differed from Mwaniki and Makokha (2013) and Abrahams et al., 2011 which reported that most SAC miss out on breakfast with its contribution being just about $10 \%$ of the recommended daily allowance intake. Findings from this study also differed from those by a review done by Sophie and Peninah (2014) on the dietary intake of school children and adolescents in developing countries that reported SAC in rural populations hardly consumed breakfast as the first meal of the day. Findings from this study can be attributed to the evidence of a breakfast campaign by the Blue Band Margarine Company at the time of data collection that encouraged the use of blue band margarine as the preferred bread spread and additional accompaniment during meals consumed at breakfast. This shows how the influence of nutrition education goes in changing attitudes and perceptions related to food among children of this age.

\section{Conclusion}

The researcher found out that the pupils had inadequate dietary intakes with low dietary diversity scores. This agrees to similar studies done on the same population that noted the inadequate and low diverse diets among the school aged population. The dietary intake of the pupils was mainly determined by the parents of the pupils as they consumed at least two meals in a day at a home set up. This shows the important role that parents play with regards to the dietary intake of their children. With regards to the snacks and lunch time meals, the pupils had the power to choose which foods to consume as they could buy the foods from the money given by their parents. Pupils therefore need to be educated on the importance of making good dietary choices of the foods they consume.

\section{Recommendations}

Nutrition education should be included in the school curriculum as school settings are ideal in promoting lifelong eating habits and lifestyles. Implementation of school health and nutrition programmes in schools should be done to ensure healthy school aged children. Also, the government should ensure implementation of the National School Meals and Nutrition Strategy to promote nutrition of school children. School meals should be included in the national and county budgets of each financial year. This will promote the school attendance and school performance of the pupils as it is a safety net for them. Further studies should be conducted on the role of parents in children's dietary choices.

\section{References}

i. Abrahams et al., (2011). Diets and mortality rates in Sub-Saharan Africa: Stages in the nutrition transition. BMC Public Health. Article number 801

ii. Barooh, P. (2012). Adolescents' Nutrition, Attitudes and Practices. IJCAES Special Issue on Basic, Applied \& Social Sciences, 2, 308-1.

iii. Bandura, A. (2006). Adolescent development from an agentic perspective. In F. Pajares \& T. Urdan (Eds.). Selfefficacy beliefs of adolescents, (Vol. 5., pp. 1-43). Greenwich, CT: Information Age Publishing 
iv. Cora Best, Nicole Neufingerl, Laura van Geel, Tina van den Briel, and Saskia Osendarp. (2010). Nutrition status of school aged children. Food and Nutrition Bulletin, vol. 31, no. 3. The United Nations University

v. Doku D, Koivusilta L, Raisamo S, Rimpela A. (2013). Socio-economic differences in adolescents' breakfast eating, fruit and vegetable consumption and physical activity in Ghana. Public Health Nutr; 16: 864-872

vi. El-Kassas G. and Ziade M. (2017). The Dual Burden of Malnutrition and Associated Dietary and Lifestyle Habits among Lebanese School Age Children Living in Orphanages in North Lebanon. Journal of Nutrition and Metabolism doi 10.1155/2017/ 4863431

vii. First Bungoma County Integrated Development Plan 2013-2017. Bungoma County Government press 2013.

viii. FAO (2011). Guidelines for measuring household and individual dietary diversity. Rome, Italy.

ix. Food and Agriculture Organization (FAO). (2004). Dietary reference intakes. Retrieved from http:/ / www.sochinut.cl/ pdf/ Recomendaciones/ DRISummaryListing.pdf

x. Gharib N, Rasheed P. (2011). Energy and macronutrient intake and dietary pattern among school children in Bahrain: a cross-sectional study. Nutr J 2011; 10: 1-12.

xi. Granner ML, Sargent RG, Calderon KS, Hussey JR, Evans AE, Watkins KW: Factors of fruit and vegetable intake by race, gender, and age among young adolescents. J Nutr Educ Behav 2004; 36: 173-180

xii. Grosvenor B. and Smolin A. (2009). Visualizing Nutrition: Everyday Choices. Pediatric Books 124:e362-e370

xiii. Kiragu et al., (2015). Nutrition knowledge, attitude and practices among urban primary school children in Nairobi City, Kenya: a KAP study. BMC Nutrition. Kiragu et al. BMC Nutrition (2015) 1:44. DOI 10.1186/ s40795-015-0040-8

xiv. Lazzeri G, Pammolli A, Azzolini E, Simi R, Meoni V, de Wet D, et al. Association between fruits and vegetables intake and frequency of breakfast and snacks consumption: a cross-sectional study. Nutr J 2013; 12:123

xv. Mbithe Dorcus David, Judith Kimiywe and Judith Waudo. (2012). Nutritional knowledge, attitude and practices and nutritional status of school-going children in Machakos District, Kenya. Nutrition and Food Sciences IACSIT Press. Singapore. 2012. Volume 39: 147-152

xvi. Ministry of Health (2010). Kenya National Clinical Nutrition and Dietetics Reference Manual. 1 st edition.

xvii. Mitchikpe CE, Dossa RA, Ategbo EA, Van Raaij JM, Kok FJ (2009). Seasonal variation in food pattern but not in energy and nutrient intakes of rural Beninese school-aged children. Public Health Nutr 2009; 12: 414-422

xviii. Mukherjee R et al. (2017). A study of the dietary habits of school children in Pune city, Maharashtra, India. Int J Community Med Public Health. Feb; 4(2):593-597

xix. Mwaniki E. W. and Makokha A. N. Nutrition Status and Associated factors among children in Public primary schools in Dagoretti, Nairobi, Kenya. (2013). African Health Sciences; 13 (1): 39-46

xx. Nago ES, Lachat CK, Huybregts L, Roberfroid D, Dossa RA, Kolsteren PW: Food, energy and macronutrient contribution of out of-home foods in school-going adolescents in Cotonou, Benin. Br J Nutr 2010; 103: 281-288

xxi. Opini B. Ruth. (2017). Nutrition Status and Associated Factors among School Going Children aged 4-10 years in Kisii Central, Kisii County, Kenya. A Master's Thesis

xxii. Pangani I, Kipamai K, Kamau J and Onywera V. (2016). Prevalence of Overweight and Obesity among Primary School Children aged 8-13 years in Dar es salaam City Tanzania. Advances in Preventive Medicine Volume 2016, Article ID 1345017, 5 pages http:// dx.doi.org/ 10.1155/ 2016/1345017

xxiii. Photographic Food Atlas for Kenyan Adolescents (2018). The Kenya-Finland Education and Research Alliance (KENFIN-EDURA)

xxiv. Rauber F, Hoffman DJ, and Vitolo MR. (2014). Diet quality from pre-school to school age in Brazilian children: a 4year follow-up in a randomized control study. Br J Nutr 2014; 111: 499-505.

xxv. Sophie Ochola and Peninah K. Masibo. Dietary Intake of school children and adolescents in developing countries (2014). AnnNutrMetab; 64

xxvi. United Nations International Children's Fund. (2016). State of the World's Children Statistical Report; United Nations International Children's Fund: New York, NY, USA

xxvii. WHO/FAO, (2010). The Nutrition Challenge. Food Systems Solution. Rome, FAO. www.fao.org/ state-of-foodsecurity-nutrition

xxviii. World Food Programme (WFP). (2015). WFP and school meals. Retrieved from http://www.wfp.org/schoolmeals/ wfp-school-meals

xxix. Yamane Taro (1967). Statistics: An Introductory Analysis, 2nd Edition, New York: Harper and Row 